\title{
A Power Management Architecture for Sensor Nodes
}

\author{
Jens Eliasson, Per Lindgren, Jerker Delsing \\ Dept. of Computer Science and Electrical Engineering \\ Luleå University of Technology \\ Luleå, Sweden \\ jens.eliasson@1tu.se, per.lindgren@ltu.se \\ jerker.delsing@ltu.se
}

\begin{abstract}
Wireless sensor nodes are a versatile, generalpurpose technology capable of measuring, monitoring and controlling their environment. Even though sensor nodes are becoming ever smaller and more power efficient, there is one area that is not yet fully addressed; Power Supply Units (PSUs). Standard solutions that are efficient enough for electronic devices with higher power consumption than sensor nodes, such as mobile phones or PDAs, may prove to be ill suited for the extreme low-power and size requirements often found on wireless sensor nodes. In this paper, a system-level design of a Power Management Architecture (PMA) is presented. The PMA is an integration of PSU hardware and various software components, and is capable of supplying a sensor node with energy from multiple sources, as well as providing status information from the PSU. The heart of the architecture is a context- and power-aware Task manager, which controls when the nodes low-power modes are activated, and is highly integrated with PSU hardware as well as other software components in the system. Its main responsibility is to schedule when energy consuming tasks can be dispatched. Depending on the task priority and system configuration, a task can be either dispatched, discarded or delayed. This approach ensures that only critical tasks will be allowed to use the battery, and that the system will be powered by renewable energy when performing other non-critical tasks.
\end{abstract}

\section{INTRODUCTION}

Sensor nodes are becoming a versatile general-purpose technology capable of measuring, monitoring and controlling their environment. These nodes are usually capable of wireless communication, and can create clusters, known as Wireless Sensor Networks (WSNs). WSNs have the potential to be deployed in even the harshest environments, e.g. in natural disaster areas, fires or other dangerous situations. Sensor networks are also suitable for long-term environmental monitoring. A great deal of research, both in the academic world as well as by the industry, have been targeted various issues; power consumption [1], [2], data aggregation [3], multi-hop routing [4], and middleware [5], [6]. But even though sensor nodes are becoming ever smaller and more power efficient, and that the routing problems for small and large sensor networks are being addressed by a large community, there is one area that is not yet fully addressed; power supplies for low-power sensor nodes. Section II, provides an overview over related work, and shows that power electronics, such as energy storage units and energy harvesters, cannot be miniaturized in the same degree

\author{
Simon J. Thompson, Yi-Bing Cheng \\ Dept. of Materials Engineering \\ Monash University \\ Melbourne, Australia \\ simon.thompson@eng.monash.edu \\ yibing.cheng@eng.monash.edu
}

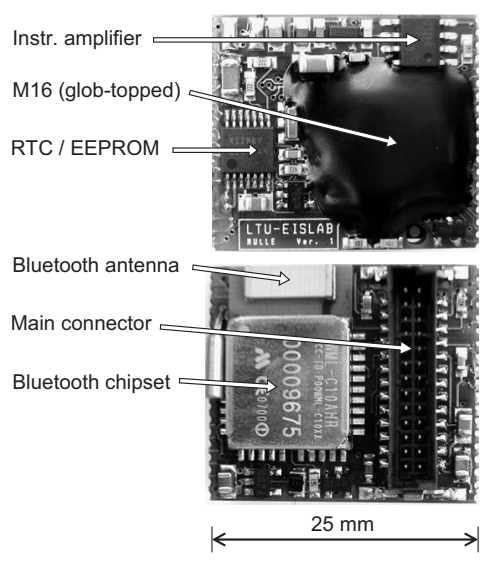

Fig. 1. MULLE overview

as other circuits [7]. The quiescent current by the PSU itself can be multitudes higher than other components of a sensor node. Microcontrollers and radios have a number of power reducing modes, which when applied, can reduce the quiescent current down to the $\mu \mathrm{A}$ range. We seek an architecture, which can efficiently provide a sensor node with fixed voltage(s), and support currents in the $\mu \mathrm{A}$ range (node in sleep mode), to tens or even hundreds of mA's (with microcontroller, radio and sensor(s) active). Section III, presents a few usage scenarios with their power and latency characteristics. The architecture must also have the ability to monitor each part of the PSU; e.g. batteries, supercapacitors, solar panels and boost converters. Section IV, outlines our design considerations and gives an overview of the requirements, such as ease of integrating the PSU with existing node hardware, size constraints, number of I/O's required to interface the PSU, price, and which information it can provide to its host node. This information must be possible to retrieve without a high overhead in energy consumption, and it should also be sufficient accurate in terms of measurement errors to support the energy-aware operations.

The sensor node used for demonstrating the proposed architecture is the MULLE [8], which for the purpose of this project was equipped with either silicon or dye sensitized photovoltaic cells. The cells, with their characteristics, are presented in 
Section V. Test setup and results are found in Section VI, while Section VII and VIII contains conclusions and future work, respectively.

\section{BACKGROUND AND RELATED WORK}

Many of the widely used sensor platforms today are designed to be used with a single energy source, namely batteries. Since batteries are the optimal technology today for energy storage, this is not a surprising fact. Battery manufacturers are competing to push the limits for reduced size and higher capacity further. Saft Technologies [9] has released the LM 33600 , which is a primary $\mathrm{Li}-\mathrm{MnO}_{2}$ battery. It can deliver an impressive $10500 \mathrm{mAh}$ at $3.0 \mathrm{~V}$, with a diameter of 33.7 $\mathrm{mm}$ and a length of $61.5 \mathrm{~mm}$, weighting just 116 grams. GP Batteries [10], has rechargeable AA sized NiMH batteries, capable of delivering up to $2700 \mathrm{mAh}$ at $1.2 \mathrm{~V}$, with up to a 1000 recharge cycles. Even though these batteries can power a wireless sensor node for months or even years, they are relatively large compared to some common sensor nodes [11], [12], and they will eventually be depleted. However, for many situations, the size of the sensor platform is not critical, and high-capacity batteries are an excellent design choice. In these cases, it is enough to have efficient voltage regulator(s) to provide the node with fixed voltage(s). Many commercial and academic sensor nodes fall into this category [12], [2]. There are however, some very interesting design approaches for devices based on energy harvesting. One of the most known systems is the Heliomote [7] from CENS [13], which is a modified Mote platform equipped with NiMH batteries and solar panels for energy scavenging. Another platform is the DuraNode [14], which uses a combination of solar panels, a windmill and rechargeable batteries as energy sources. However, the small form factor of the MULLE platform used in this work, prohibited the use of such large solar panels and energy storage devices found in both the Heliomote and DuraNode platforms. Where these platforms have solar panels capable of delivering over $120 \mathrm{~mA}$, our platform is limited to a solar panel capable of delivering only one tenth of the current, approximately $12 \mathrm{~mA}$. Our platform also has a total storage capacity of $2 \mathrm{~F}$, while the Heliomote has NiMH batteries and the DuraNode holds a large 100 F capacitor. Both the Heliomote and the DuraNode utilizes customized low-power radios, while the MULLE use TCP/IP and Bluetooth for improved interoperability with computers, mobile phones and PDAs.

\section{SCENARIOS AND REQUIREMENTS}

To be able to design the architecture for a large number of scenarios with quite different low-power requirements, the scenarios were divided into several classes. The classes are mainly categorized by their maximum operating lifetime and desired communication latency. The term latency is defined as the maximum time it can take for a user, either human or machine, to successfully make a connection to a sensor node.

\section{A. Scenario Classes}

The MULLE, see Fig. 1, system has successfully been used in a number of projects, ranging from monitoring of elderly to deployment on cross-country skiers during a cold Swedish winter. A classification was needed to be able to identify both soft and hard requirements of the most commonly used scenarios. All these usage scenarios have different requirements on latency and power consumption, and were divided into three classes.

- Short-time, low latency. This class has the following requirements; the sensor node should only live for a limited time (ranging from hours up to a few days), and transmit its data frequently, e.g. multiple transmissions every hour or even continuously. Typical scenarios include sport events and patient monitoring, where a user can replace empty batteries easily.

- Medium-time, variable latency. Scenarios in this class can require a sensor node to perform measurements and transmission for a number of months. The latency is not critical, and transmissions can occur up to a few times per day. This scenario includes for example using GPS to monitor the position of a boat during summer, or keeping track of the temperature of a summer house during winter. A user can here replace batteries typically once per year.

- Long-time, unimportant latency for data. The famous Great Duck Island monitoring experiment [15] falls into this class. Other sensor types can include fire alarms or scenarios where it is difficult for users to replace empty batteries because of the nodes location. The requirement for this class is that the sensor node should live for more than a few years, or even as long as possible. Data transmission occurs when data buffers starts to fill up, or when the sensor node registered a critical event (e.g. a fire alarm). Even though radio transmissions must be avoided to conserve energy, nodes should be configured to periodically transmit keep-alive messages so that users know if nodes are performing their tasks or if they have malfunctioned.

The need of a sophisticated PSU in the first class is not as important as in the two latter. With knowledge of the expected system lifetime and latency requirements, the required battery capacity can easily be calculated if the system power consumption is known. When designing for the second class, the PSU should provide the sensor node with (at least) the battery status. In the third class, the PSU must try to prolong the lifetime, while still meeting latency requirements (such as the maximum interval for keep-alive messages). The PSU must also take into consideration that the available renewable energy can vary over time.

\section{DESIGN CONSIDERATIONS}

The MULLE embedded system [8], which was used as a prototype platform during the making of this paper, is a small Bluetooth-enabled sensor node. Typical usage scenarios include patient monitoring, sport events and other situations 


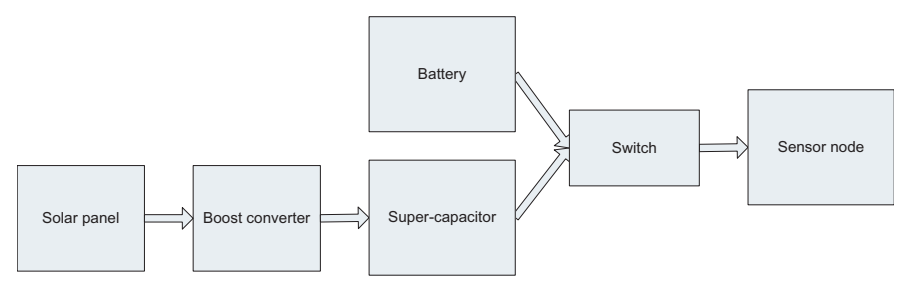

Fig. 2. Power Supply Unit

where data from sensor(s) should be transmitted to the Internet or into a local network using TCP/IP. To enable the MULLE to reach a system lifetime in the range of months or even years, an energy harvesting strategy using photovoltaic cells was chosen. The MULLE size is approximately $4 \mathrm{~cm}^{2}$, and hence the PSU should be in the same order of size. The PSU size constraints was set so that the solar panels should not be larger than $8-10 \mathrm{~cm}^{2}$, i.e. not to increase the area with more than approximately $100 \%$. Ideally, the solar panels should match the size of the MULLE, see Fig. 1. The hardware design should provide a common base, while allowing software modifications to optimize the behavior of the PSU for different application scenarios. The PSU should support a variety of different battery types, such as Lithium, Lithium-Ion and $\mathrm{NiMH}$, and also handle energy harvesting devices, such as photovoltaic cells (solar cells).

\section{A. Hardware}

The PSU hardware consists of several components, see Fig. 2. The heart of the system is an Analog Devices ADM691A [16] switch, that selects which energy source to use. The ADM691A has two inputs and hence supports two energy sources. The switch is connected to a Lishen SP0425AB [17] Li-Ion Polymer battery and an energy harvesting system. The latter system consists of solar panels, with either silicon or dye sensitized cells. The cells are in turn connected to a high-efficiency Maxim MAX867 [18] boost converter, which produces a fixed $4.5 \mathrm{~V}$ output. A low-power comparator monitors the output voltage from the cells, and when the level is higher than the minimum startup voltage (approximately 1.1 $\mathrm{V})$, the boost converter is activated. The task of the converter is to ramp up the voltage to $4.5 \mathrm{~V}$ (the maximum input level of the MULLE) and thereby to charge two supercapacitors of $1 \mathrm{~F}$ each.

$$
E=\frac{C V^{2}}{2}
$$

These supercapacitors can hold a substantial amount of energy, see Eq. 1, and are insensitive for over- and undercharge, and can be recharged virtually an unlimited number of times. Batteries however, are more or less sensitive to improper charge currents and have a limited number of recharge cycles. The capacitors do however suffer from a high leakage current, i.e. they can be drained in less than $24 \mathrm{~h}$. without any load. The hardware also includes a number of analog and digital signals, which are used to read status information. The information

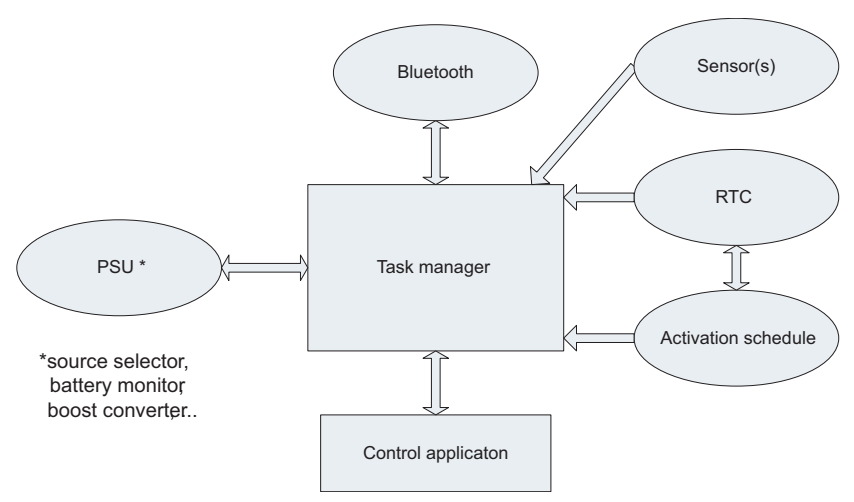

Fig. 3. Power Management Architecture

includes voltages over the solar panels, output voltage of the boost converter and the battery voltage. Digital signals are used to give interrupts, e.g. when the boost converter is activated or when the switch changes source. Analog signals are sampled using the microcontroller's A/D-converter and a DS2783 $\mathrm{I}^{2} \mathrm{C}$ battery monitor chip [19], while a MAX7315 I ${ }^{2} \mathrm{C}$ port expander [20] are used for digital signals. The use of an $\mathrm{I}^{2} \mathrm{C}$ bus minimizes the number of $\mathrm{I} / \mathrm{Os}$ that are required for interacting with the PSU. To minimize the power consumption, a design choice was made to place the boost converter in front of the supercapacitors. This way, the converters high current consumption of $100 \mu \mathrm{A}$, will be drawn when the capacitors are being charged, and not when they are being used as a source. And by placing the boost converter in front of the supercapacitors, it will also act as a buffer; ensuring that the solar panels operates at a good bias point. When the MULLE is powered by the solar panels, a MOSFET transistor is used to disconnect the battery from the ADM691A switch. This reduces the current, taken from the battery, from 940 $\mu \mathrm{A}$ to $72.5 \mu \mathrm{A}$ (which is consumed by the battery monitor chip). During our experiments, the most important goal was to monitor all voltages, however in real world applications, the MOSFET will be placed so that it disconnects the battery monitor as well, further minimizing the current drawn from the battery. All current measurements were performed with a Keithly 6485 Picoammeter.

\section{B. Software}

The software supports a number of low-power modes, as well as monitoring of the PSU hardware. The Task manager, see Fig. 3, receives information from the power supply; e.g. battery levels and status of the energy harvesting system. It also receives requests from energy demanding tasks, such as turning on a sensor or making a data transmission. Depending on the priority of the task, and the available energy levels; the task can be dispatched, delayed or discarded. The estimated energy required for each task must be given at compile time, and is obtained by characterization techniques [21]. The Task manager also has a configuration which is given at compile time, which sets e.g. priority levels (i.e. which level that is allowed to use the battery), and what kind if actions that are 


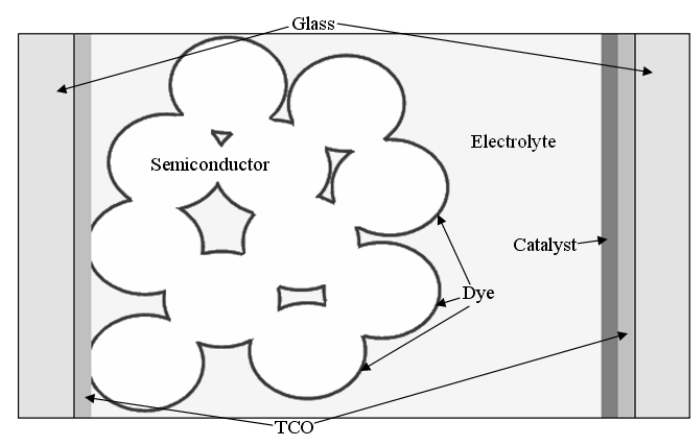

Fig. 4. Dye sensitized solar cells

to be performed when a task request is received. The Task manager acts as a context- and energy-aware task scheduler, controlling when energy consuming tasks are allowed to be dispatched.

\section{Power consumption model}

The power consumption can be described with Eq. 2, and depends on the node power consumption in sleep mode together with radio activity consumption. The consumption caused by the wireless system can be calculated by the frequency of activations, either in listening mode or outgoing connections.

$$
\begin{gathered}
E_{\text {node }}=T \times\left(P_{\text {sleep }}-P_{\text {harvest }}\right)+E_{b t} \\
E_{\text {bt }}=f_{\text {listen }} \times E_{\text {listen }}+E_{\text {incon }}+f_{\text {con }} \times E_{\text {outcon }}
\end{gathered}
$$

Together with Eq. 2, the activation schedule and the Task manager rules, a node can during runtime estimate the remaining lifetime. It can also compare energy levels with the model to see if the node is using more energy than estimated.

\section{PHOTOVOLTAIC CELLS}

Dye sensitized solar cells (DSSCs) [22] are currently being investigated by numerous groups around the world as a low cost alternative to amorphous silicon photovoltaic cells. In low light applications, DSSCs outperform silicon solar cells and have been seen to match or outperform silicon in large scale outdoor applications. As a result DSSCs are expected to find a market as an inexpensive portable power source for low powered devices such as sensor networks.

A typical dye sensitized solar cell, Fig. 4, comprises of a pair of flat glass electrodes whose surface is coated by a transparent conductive oxide layer (TCO). On one of these electrodes (the working electrode) is a film of a nanoporous semiconductor, usually titanium dioxide $\left(\mathrm{TiO}_{2}\right)$, which is coated by a layer of light absorbing (ruthenium complex) dye. Between the electrodes is an electrolyte, which consists of a redox couple, usually iodide/triiodide $\left(\mathrm{I}^{-} / \mathrm{I}_{3}{ }^{-}\right)$, in an organic solvent. The other electrode (counter electrode) has a thin layer of platinum to catalyse the regeneration of the electrolyte.
The process for electricity generation is as follows. Light is absorbed by the dye, exciting an electron in the ruthenium, which is then transferred into the conduction band of the $\mathrm{TiO}_{2}$. The dye has been left in an oxidised state. The electron is transported to the electrode via the semiconductor film and through the load circuit. The process is completed by the redox couple which is reduced at the counter electrode, catalysed by the platinum layer, converting $\mathrm{I}_{3}{ }^{-}$to $\mathrm{I}^{-}$, and is subsequently oxidized by the dye, which returns the dye to its ground state and converts $\mathrm{I}^{-}$back to $\mathrm{I}_{3}{ }^{-}$. The voltage produced is determined by the potential difference between the redox couple and the conduction band of the semiconductor, and the current is determined by the number of photons absorbed by the dye. This power generation methods differs from silicon solar cells which absorbs photons which have more energy than the band gap of the semiconductor. In DSSCs, light is absorbed by the dye, which transfers an excited electron to the semiconductor. The advantage of this is that the absorption spectrum is reliant on the dye and not the band gap of the semiconductor, making it easier to tune the spectral harvesting of light. This property could be used to create transparent power harvesting windows for skyscrapers, etc [23]. Another advantage of the DSSC is that the components are cheap. Instead of having a single crystal of silicon, a DSSC typically consists of nanoparticles of $\mathrm{TiO}_{2}$, a readily available material, already used in many applications. These applications include white house paint and toothpaste, as well as more novel applications such as self cleaning glass and air filtration. During operation DSSC are not sensitive to elevated temperatures experienced in some operating environments, conversely a silicon solar cells performance decays as the temperature increases.

Currently the weakness of the DSSC is the electrolyte used in the regeneration of the dye. The volatile and corrosive nature of this substance makes sealing of cells difficult and greatly reduces the lifetime of the cell. To overcome this problem there is research into many alternate electrolytes. These include: ionic liquids [24], plastic crystals [25] and solid state electrolytes [26]. Currently none of these alternatives are as efficient as the liquid electrolyte currently employed.

The DSSCs used were made at Monash University. The components used were P25 (Deguassa) $\left(\mathrm{TiO}_{2}\right)$, tech 16 FTO glass (Hartford), N719 dye $\left(\left(\mathrm{nBu}_{4} \mathrm{~N}\right)_{2}\left[\mathrm{Ru}(\mathrm{Hdcbpy})_{2}(\mathrm{NCS})_{2}\right]\right)$ where dcbpy is 4,4-dicarboxylate-2,2-bipyridine) and the electrolyte consisted of; $0.6 \mathrm{M}$ tetrabutylammonium iodide (Aldrich), 0.5 M LiI (Lancaster), $0.04 \mathrm{M} \mathrm{I}_{2}$ (Lancaster) and $0.05 \mathrm{M}$ 4-tert-butylpyridine (Aldrich) in acetonitrile.

\section{TEST RESULTS}

The test setup consisted of one MULLE system, a Bluetooth access point (D-Link DBT900-AP [27]), one workstation acting is a NTP server and a laptop for logging purposes. When the MULLE was turned on, it connected to the access point. After an IP-address was obtained using DHCP, the NTP protocol [28] was used to synchronize the MULLE Realtime Clock (RTC). After all these stages were performed, 


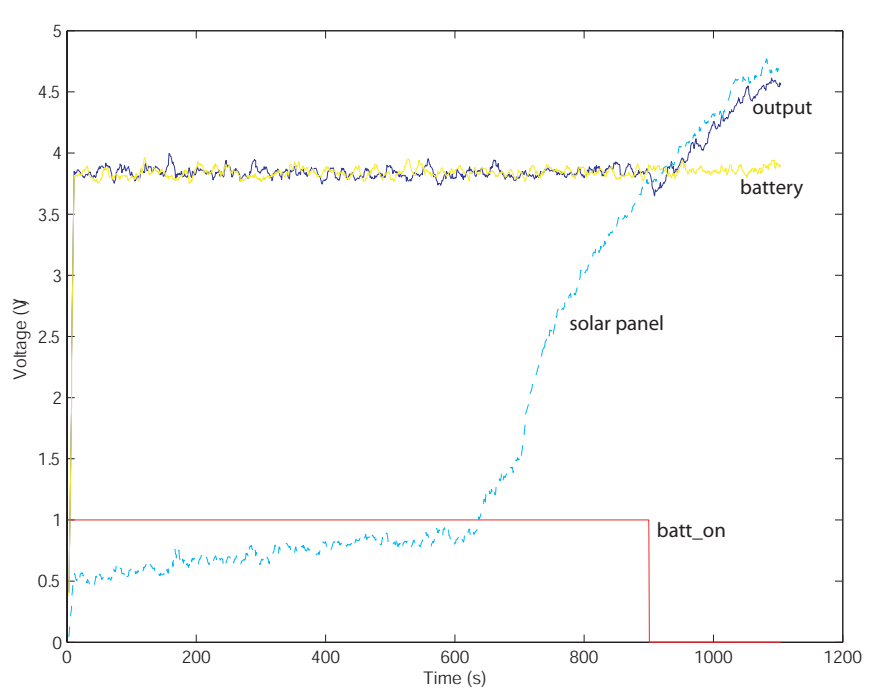

Fig. 5. Solar panel charge

the MULLE disconnects from the access point and starts to execute a duty-cycle based operating mode called TimeSynchronous [29]. It uses an activation schedule to periodically enable the Bluetooth module to listen for incoming connections. This listening mode increases the current consumption from $100 \mu \mathrm{A}$ to $1 \mathrm{~mA}$, causing the supercapacitors to drain at a faster rate. The activation schedule, which is provided by the user, either at compile time or dynamically during runtime, must be adjusted to suite the solar panels. All tests utilized a schedule which caused the MULLE to activate its Bluetooth module for a 2 minute period, every 20 minutes between 08.00 and 17.00.

All tests were performed outdoors during late summer in Luleå (lat. 65.61725, lng. 22.13795), northern Sweden. A data acquisition system, with four 10-bit inputs, was used to monitor the PSU. Data from the acquisition system was sent to the laptop for further processing and logging purposes. All results and pictures were generated using MATLAB. All tests were performed during the end of August. Two different types of solar panels were used, one 6-cell silicon panel capable of producing $4 \mathrm{~V}$ open-circuit-voltage (OCV) and $12 \mathrm{~mA}$ shortcircuit-current (SSC) and one dye-sensitized cell capable of $3 \mathrm{~V} \mathrm{OCV}$ and $1 \mathrm{~mA} \mathrm{SSC}$. Due to the sealing problem with the DSSCs, the $10 \mathrm{~cm}^{2}$ 6-cell silicon solar panel was used for long term tests.

\section{A. Supercapacitor charge without load}

As shown in Fig. 5, the system is powered by the battery, and that the PSU indicator for battery usage (batt_on) is high. 600 seconds after the solar panel was attached, the input voltage of the boost converter reached $1.1 \mathrm{~V}$, and caused the comparator to activate the boost converter to charge two $1 \mathrm{~F}$ supercapacitors. After another 250 seconds, when the voltage over the capacitors reaches the battery voltage, the ADM691A indicates (with the batt_on signal) to the MULLE that it is now powered by the solar panels and switches from the battery to

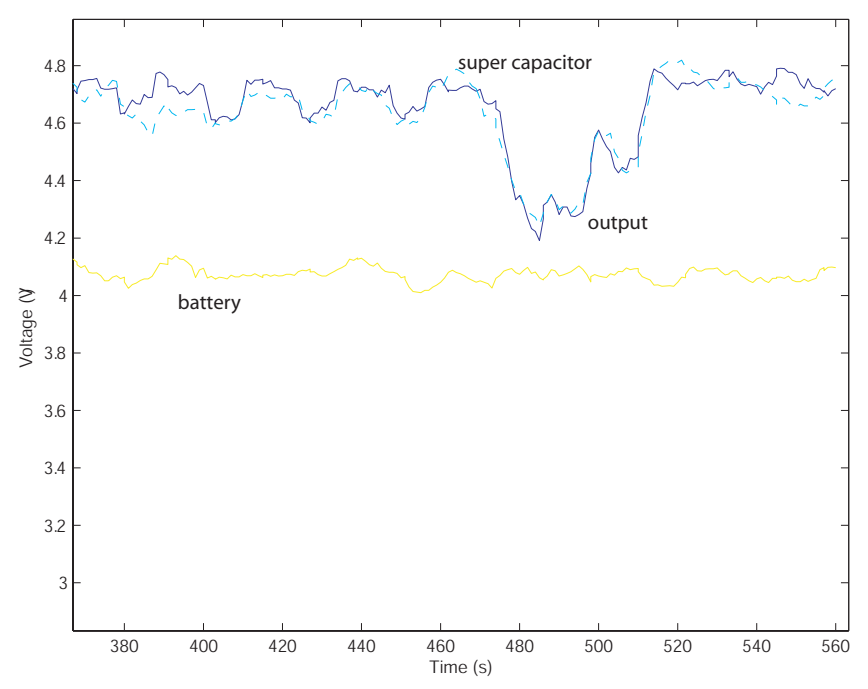

Fig. 6. Connection

the supercapacitors. The voltage over the capacitors reaches $4.5 \mathrm{~V}$ after about 1100 seconds and then slowly levels out. To fully charge the capacitors takes less than 20 minutes,

\section{B. Bluetooth connection}

Fig. 6 shows an example of a performed Bluetooth connection when the system is powered by the solar panels. Approximately $480 \mathrm{~s}$ after the measurement is started and the MULLE has enabled the Bluetooth module, a Bluetooth connection was performed. After successful connection, an ICMP 'echo request', (ping) IP-packet was used to transmit data to the MULLE. After eight packets were transmitted and recieved, the connection was shut down. As indicated, the voltage over the supercapacitors is reduced from $4.6 \mathrm{~V}$ to 4.1 V. When the connection is closed, we see how the solar panels charge the supercapacitors and the voltage increases until they are fully charged again. For this experiment, a capacitor of $0.44 \mathrm{~F}$ was used to allow measurements with higher accuracy. The usage of the ping command enabled easy monitoring of packet loss and round-trip time estimation.

\section{Supercapacitor self-discharge}

Preliminary measurements indicates, see Fig. 7, that the supercapacitors are discharged in 11 hours, with a sensor node in sleep mode as load. This high discharge rate requires that the capacitors are recharged on a daily basis by the solar panels.

\section{CONCLUSION}

This paper demonstrates a power management architecture designed for wireless sensor nodes. Important design requirements are: small form factor, power efficiency, ease of integration with the host node and the possibility to monitor the behavior of the entire power supply. The proposed Task manager uses the PSU monitoring system to dynamically make decisions whether energy consuming tasks should be 


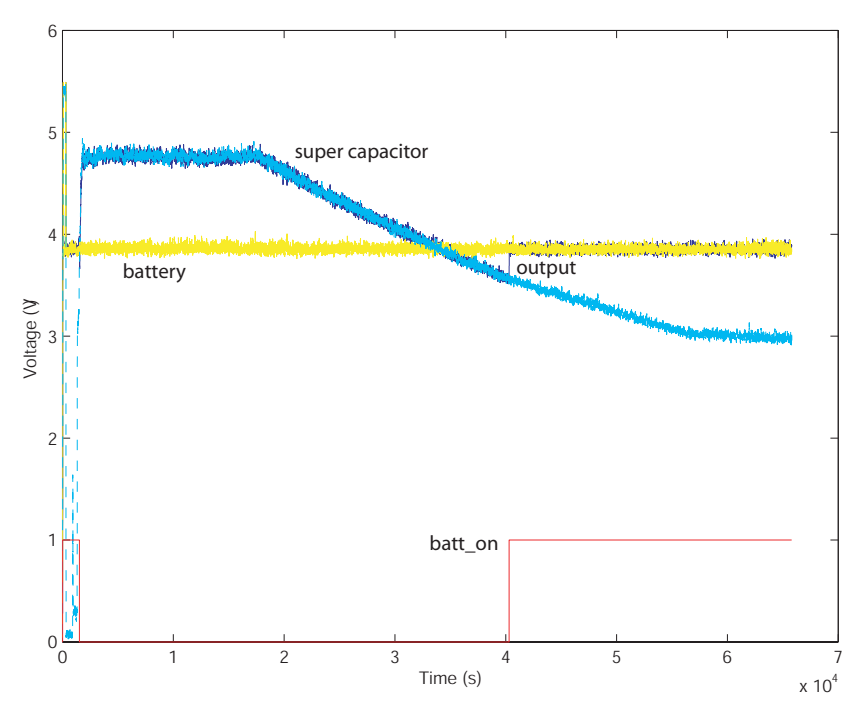

Fig. 7. PSU / Supercapacitor discharge

dispatched, delayed or discarded. If the system is running on the battery, the Task manager only allows high priority tasks to be dispatched while postponing all other tasks until the system has enough stored renewable energy. This approach ensures that the system never wastes energy on tasks that cannot be completed, and keeps the sensor node operating with a high utilization of available renewable energy. The proposed design combines PSU hardware, duty cycling and an activation schedule into an efficient power management architecture. The architecture is verified with real-world experiments, where a MULLE sensor node was equipped with solar panels. Experimental results indicate that the power consumption can be drastically reduced while maintaining system performance.

\section{FUTURE WORK}

All tests that were conducted were performed during a time span of a few hours. What needs to be investigated further is the long-term performance of the system. This is scheduled to be performed in January 2007 in Australia. The possibility to distribute the status of a sensor node's energy levels to other sensor nodes in the network were not investigated. This will enable Task managers from different nodes to communicate with each other over the network, and to distribute the workload among a number of available sensor nodes.

\section{REFERENCES}

[1] M. Lundberg, J. Eliasson, L. Svensson, and P. Lindgren, "Context Aware Power Optimizations of Wireless Embedded Internet Systems," in Proceedings of the 21st IEEE Instrumentation and Measurement Technology Conference, IMTC 04, vol. 1, Como, Italy, May 2004, pp. 91-95.

[2] J. King, R. Bose, S. Pickles, A. Helal, S. V. Ploeg, and J. Russo, "Atlas: A Service-Oriented Sensor Platform," in ACM Conference on Embedded Networked Sensor Systems (Sensys), Boulder, USA, 2006.

[3] H. Dubois-Ferrire, D. Estrin, and M. Vetterli, "Packet combining in sensor networks," in Conference On Embedded Networked Sensor Systems, San Diego, USA, 2004.
[4] J. Polastre, J. Hill, and D. Culler, "Versatile low power media access for wireless sensor networks," in Conference On Embedded Networked Sensor Systems, Baltimore, USA, 2004.

[5] E. Souto, G. Guimares, G. Vasconcelos, M. Vieira, N. Rosa, and C. Ferraz, "Middleware challenges for wireless sensor networks," in Proceedings of the 2nd workshop on Middleware for pervasive and adhoc computing MPAC, 2004.

[6] K. Terfloth, G. Wittenburg, and J. Schiller, "FACTS - A Rule-based Middleware Architecture for Wireless Sensor Networks," in Communication System Software and Middleware, Comsware, 2006.

[7] A. Kansal, D. Potter, and M. Srivastava, "Performance Aware Tasking for Environmentally Powered Sensor Networks," in Joint International Conference on Measurement and Modeling of Computer Systems, New York, NY, USA, 2004, pp. 223 - 234.

[8] J. Johansson, M. Völker, J. Eliasson, Å. Östmark, P. Lindgren, and J. Delsing, "MULLE: A Minimal Sensor Networking Device - Implementation and Manufacturing Challenges," in IMAPS Nordic 2004, Helsing"or, Denmark, September 2004, pp. 265-271.

[9] "Saft batteries," http://www.saftbatteries.com/, September 2006.

[10] "GP Batteries," http://www.gpbatteries.com/, September 2006.

[11] "A Distributed Environment for Prototyping Ad Hoc Networks," September 2006.

[12] "Crossbow Inc." http://www.xbow.com/, September 2006.

[13] "Center for Embedded Networking Senses," www.cens.ucla.edu/, September 2006.

[14] C. Park, Q. Chou, and P. Shinozuka, "DuraNode: wireless networked sensor for structural health monitoring," in 10th SPIE International Symposium Nondestructive Evaluation for Health Monitoring and Diagnostics, San Diego, USA, March 2005.

[15] "Great duck island," http://www.greatduckisland.net/, Great Duck Island, September 2006.

[16] "Microprocessor supervisory circuits," http://www.analog.com/, September 2006.

[17] "Lishen battery ltd." http://www.lishen.com/, September 2006

[18] “Adjustable Output, Single-Cell DC-DC Converter," http://www.maximic.com/, September 2006.

[19] "Maxim/Dallas DS2783 fuel gage," http://www.maxim-ic.com/ds2783/, September 2006.

[20] “Maxim 8-port I/0 Expander," http://www.maxim-ic.com/max7315/, December 2006.

[21] M. Lundberg, J. Eliasson, J. Allan, J. Johansson, and P. Lindgren, "Power Characterization of a Bluetooth-Equipped Sensor Node," in Workshop on Real-World Wireless Sensor Networks, Stockholm, Sweden, 2005.

[22] B. O'Regan and M. Gratzel, "A low-cost, high-efficiency solar cell based on dye-sensitized colloidal Ti0 2 films," Nature, vol. 353, p. 737, 1991.

[23] M. Gratzel, "Perspectives for dye-sensitized nanocrystalline solar cells," Progress in photovoltaics, vol. 8, p. 171, 2000.

[24] H. Paulsson, L. Kloo, A. Hagfeldt, and G. Boschloo, "Electron transport and recombination in dye-sensitized solar cells with ionic liquid electrolytes," Journal of Electroanalytical Chemistry, vol. 586, pp. 56-61, 2006.

[25] Q. Dai, D. R. MacFarlane, and M. Forsyth, "High mobility $\mathrm{I}^{-} / \mathrm{I}_{3}-$ redox couple in a molecular plastic crystal: A potential new generation of electrolyte for solid-state photoelectrochemical cells," Solid state ionics, vol. 177, p. 395, 2006.

[26] U. Bach, D. Lupo, P. Comte, J. E. Moser, F. Weissortel, J. Salbeck, H. Spreitzer, and M. Gratzel, "Solid-state dye-sensitized mesoporous $\mathrm{TiO}_{2}$ solar cells with high photon-to-electron conversion efficiencies," Nature, vol. 395, pp. 583-585, 1998.

[27] "Bluetooth - LAN Access Point," http://www.dlink.co.uk/, September 2006, D-Link

[28] "RFC 1305 - Network Time Protocol (version 3)," March 2005, http://www.faqs.org/rfcs/rfc1305.html.

[29] J. Eliasson, M. Lundberg, and P. Lindgren, "Time Synchronous Bluetooth Sensor Networks," in IEEE Consumer Communications and Networking Conference (CCNC 2006), vol. 1, Las Vegas, Nevada, USA, January 2006, pp. 336-340. 